\title{
Images of the month 2: Extensive pneumocephalus after subdural haematoma
}

\author{
Authors: Vanderson Neri ${ }^{\mathrm{A}}$ and Carla Slater ${ }^{\mathrm{B}}$
}

KEYWORDS: pneumochephalus, subdural haematoma, Mount Fuji

DOI: $10.7861 /$ clinmed.2021-0548

\section{Case presentation}

An 80-year-old man presented with complaints of recurrent frontal headaches. Computed tomography (CT) of the brain showed a heterogeneous bilateral haematoma from the frontal to the occipital region (Fig 1a). Bilateral parietal craniotomy was performed. One day later, an extensive frontal pneumocephalus appeared, with compression of the bilateral cerebral parenchyma (Fig 1b). The patient remained asymptomatic, with no cognitive alterations or focal neurological deficits. Subsequent CT showed a gradual reduction of the pneumocephalus spontaneously until complete resolution, about 2 months later (Fig 1C).

\section{Discussion}

Pneumocephalus can happen after neurosurgeries like this, occurring after brain compression caused by chronic bleeding, associated with common cerebral atrophy at this age. After drainage of the haematoma, there was no cerebral expansion and the subdural space was filled by air, which occupied the space left by the blood. Characteristic radiology imaging is the 'Mount Fuji sign', a silhouette similar to the Japanese volcano of the same name. ${ }^{1,2}$.

\section{References}

1 Schirmer CM, Heilman CB, Bhadwaj A. Pneumocephalus: case illustrations and review. Neurocrit Care Soc 2010;13:152-8.

2 Michel SJ. The Mount Fuji sign. Radiology 2000;232:449-50.

Address for correspondence: Dr Vanderson Neri, Hospital Federal da Lagoa, 501 Jardim Botânico, Rio de Janeiro 22470-050, Brazil.

Email: vandersoncneri@hotmail.com

Twitter: @vcneri

Authors: A professor and consultant of neurology, Hospital Federal da Lagoa, Rio de Janeiro, Brazil; ${ }^{B}$ neurology consultant, Hospital Duque de Caxias, Rio de Janeiro, Brazil
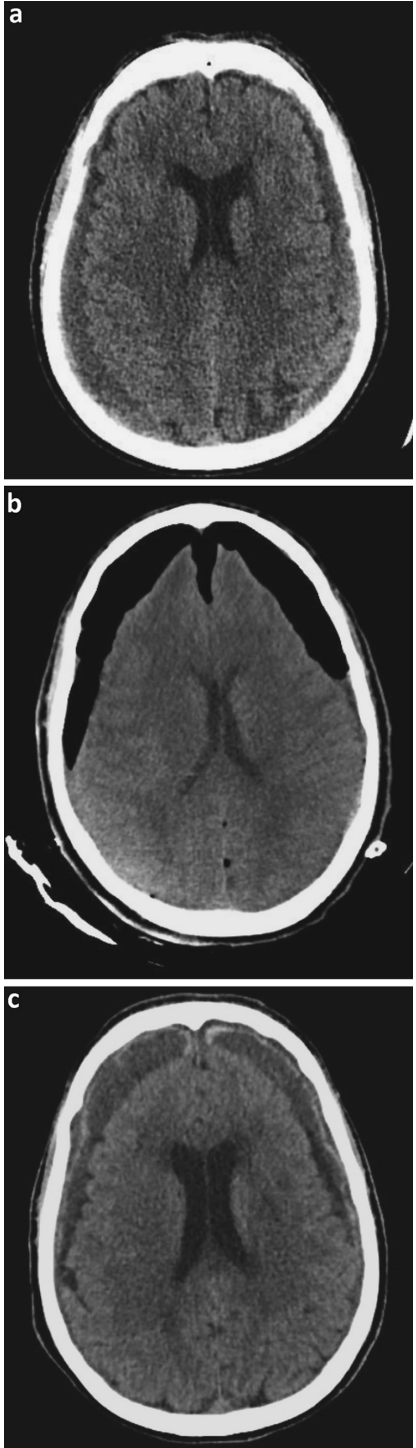

Fig 1. Computed tomography of the brain. a) Heterogeneous subdural haematoma, extending from frontal to occipital region bilaterally. b) Massive accumulation of air in the subdural space with compressive effects on both frontal lobes, the 'Mount Fuji sign'. c) Follow-up scan showing resolution of pneumocephalus. 